\title{
DESIGUALDAD Y EXCLUSIÓN SOCIAL COMO DESAFÍOS PARA LAS DEMOCRACIAS EN AMÉRICA LATINA ${ }^{1}$
}

\author{
Inequality and Social Exclusion as a Challenge for Democracies in Latin America/ \\ Desigualdade e Exclusão Social como Desafio para as Democracias na América \\ Latina
}

Betilde Muñoz-Pogossian²

\begin{abstract}
RESUMO
Nos últimos anos, os países da América Latina têm dado passos importantes na área da inclusão. No entanto, milhões de cidadãos ainda não participam, adequadamente, da vida social, económica e política das suas respectivas sociedades. O objectivo deste artigo é avaliar a relação entre a desigualdade persistente e as suas as implicações, reflectidas nas democracias, nos países da região. A metodologia utilizada no desenvolvimento deste artigo foi analítica e qualitativa, onde as contribuições de autores como Munck (2005), Casas-Zamora et al (2014), Zovatto e Herrera (2011) e dados do trabalho da CEPAL e do PNUD, entre otros, foram fundamentais para a análise incorporada no trabalho.Entre os principais aspectos avaliados, como conseqüências da desigualdade existente na América Latina, está o desencantamento da cidadania com a democracia e a erosão da sua legitimidade; o aumento da violência e outras práticas antissociais; o acesso diferenciado aos direitos, especialmente para os indígenas e afrodescendentes; o surgimento de novos conflitos, em particular das classes médias e a redução da representação e qualidade das decisões advindas do sistema político. $O$ artigo conclui que a concentração do poder económico-político em poucas mãos prejudica a prática democrática e os processos políticos, muitas vezes colocando o Estado a serviço das elites, afectando o pleno exercício dos direitos humanos, deslegitimizando a representação política e instituições republicanas.
\end{abstract}

Palavras-chave: Equidade, Inclusão Social, Acesso a Direitos, Democracia, Exclusão de Sociedades.

\footnotetext{
${ }^{1}$ Trabajo originalmente preparado para la conferencia "Los Dilemas de la Democracia en América Latina," organizado por FLACSO México y el Instituto de Investigaciones Jurídicas de la UNAM, México, DF, septiembre 30, octubre 1-2, 2015.

${ }^{2}$ Directora del Departamento de Inclusión Social de la Secretaría de Acceso a Derechos y Equidad de la Organización de los Estados Americanos (OEA). Ph.D. en Ciencias Políticas y Master en Relaciones Internacionales. Durante su trayectoria internacional de más de 15 años, se desempeñó como Directora del Departamento de Observación y Cooperación Electoral de la OEA, como Oficial de Políticas Senior de la Secretaría de Asuntos Políticos de la misma organización, y ha sido observadora electoral internacional en más de 50 misiones electorales de la OEA. Actualmente se dedica a temas vinculados a la desigualdad política y social en América Latina desde un enfoque de derechos, y sus implicaciones para la democracia y los derechos humanos en la región. Washington, USA. Correo electrónico: bmunoz@oas.org; bvmr@hotmail.com
} 
Desigualdad y exclusión social como desafíos para las democracias en América Latina.

Recebido: Junho 2018 Aceitado: Julho 2018

RESUMEN

En los últimos años, los países de América Latina han dado importantes pasos en materia de inclusión. Sin embargo, millones de ciudadanos y ciudadanas aun no participan adecuadamente de la vida social, económica, y política de sus respectivas sociedades. El objetivo de este trabajo es evaluar la relación entre la persistente desigualdad, y las implicaciones que esto tiene para las democracias en los países de la región. La metodología empleada en el desarrollo de este artículo fue analítica y cualitativa, donde las contribuciones de autores como Munck (2005), Casas-Zamora et al (2014), Zovatto \& Herrera (2011) y datos provenientes de trabajos de CEPAL y PNUD, entre otros, fueron clave para el análisis plasmado en el trabajo. Entre los principales aspectos evaluados como consecuencias de la desigualdad existente en América Latina se encuentran (a) el desencanto de la ciudadanía con la democracia, y erosión de su legitimidad, (b) el aumento de la violencia y otras prácticas antisociales, (c) el acceso diferenciado a derechos, especialmente para indígenas y afrodescendientes, (d) la emergencia de nuevas conflictividades, en particular proveniente de las clases medias, y (e) la reducida representatividad y calidad de las decisiones provenientes del sistema político. El artículo concluye que la concentración del poder económico-político en pocas manos desvirtúa la práctica democrática y los procesos políticos, con frecuencia poniendo al Estado al servicio de las élites, afectando el ejercicio pleno de los derechos humanos, deslegitimando la representación política, y las instituciones republicanas.

Palabras clave: Equidad, Inclusión Social, Acceso a Derechos, democracia, sociedades excluyentes.

\begin{abstract}
In the last few years, Latin American countries have made great strides in terms of inclusion. However, millions of Latin Americans still do not fully participate in the social, economic and political life of their societies. The objective of this paper is to evaluate the relationship between the persistent levels of inequality in the region, and the implications for democracy. The methodology utilized for the paper was analytical and qualitative, considering contributions by authors such as Munck (2005), Casas-Zamora et al (2014), Zovatto \& Herrera (2011) as well as data from ECLAC and UNDP, among others. Some of the key aspects evaluated in this analysis include: (a) citizens' disenchantment with democracy, and erosion of democratic legitimacy, (b) the increase of violence and other anti-social practices, (c) the differentiated access to rights, specially for indigenous peoples and afrodescedants, (d) the emergence of new forms of conflict, specially from the middle class, and (e) the reduced legitimacy and representativeness of the decisions emerging from the political system. The article concludes that the concentration of economic and political power in the hands of a few undermines democratic practice and political processes, often putting the State at the service of
\end{abstract}


elites, affecting the full exercise of human rights, delegitimizing political representation as well as republican institutions.

Key Words: Equity, Social Inclusion, Access to Rights, democracy, Excluding societies.

\section{INTRODUCCIÓN}

Durante los últimos años, los Estados Latinoamericanos han tomado medidas de política pública para generar mayor inclusión y una mejor redistribución del goce de los beneficios del crecimiento económico, motivados, en gran parte, por el alto precio mundial de las materias primas. De igual forma, en materia de democratización y ejercicio de los derechos políticos, se han dado progresos considerables en los últimos quince años. Como resultado, hoy los países de Latinoamérica y el Caribe son más estables, cuentan con instituciones más sólidas y, a pesar de algunos retrocesos, gobiernos más democráticos. Bajo este proceso de consolidación, la celebración periódica de elecciones ha sido un factor vital por medio del cual se expresa y legitima la voluntad popular. Así, las elecciones se han afianzado como el único medio legítimo de acceso al poder.

Sin embargo, los desafíos continúan. A pesar de estos avances, los ciudadanos latinoamericanos están convencidos de que sus países son desiguales y que la mayor parte de la riqueza está concentrada en pocas manos. Además, asocian esa desigualdad a la incapacidad de los partidos y de las diversas instituciones del Estado (Ejecutivo, Judicial y Legislativo) para procesar sus demandas. Esa desigualdad percibida por los ciudadanos sobre la manera inequitativa en la que se distribuyen los bienes económicos, políticos y sociales se correlaciona con la desconfianza hacia las instituciones y los actores políticos, lo cual dificulta la salud de la democracia o genera "democracias de baja calidad" (O’Donnell, 1998). Ante este escenario de avances pero retos persistentes, el presente trabajo ofrece un balance sobre las implicaciones de la desigualdad y la persistencia de sociedades excluyentes para los procesos democráticos en América Latina. 
Desigualdad y exclusión social como desafíos para las democracias en América Latina.

Así, en una primera parte, se documentan los avances regionales desde dos aristas: en materia de inclusión social y en materia de consolidación democrática. En la segunda parte se apuntan los principales desafíos que aún enfrentan los países de la región, haciendo especial hincapié en los vínculos estrechos y perniciosos entre la desigualdad y los desafíos de la democracia. En la última sección, se ofrecen algunas reflexiones sobre la situación de América Latina y su condición de ser "la región más desigual del mundo." En el estudio se argumenta que la concentración de poder económico-político desvirtúa la práctica democrática y los procesos políticos.

\section{AVANCES REGIONALES EN MATERIA SOCIAL Y DEMOCRÁTICA}

El debate sobre la desigualdad es esencialmente político (Oxfam, 2015). Está ilustrado por la tendencia reciente de introducir la cuestión social en el centro de la agenda política de América Latina y el Caribe, región democratizada que se ubica en posiciones ejemplares en los índices que miden el nivel democrático (Wehr, 2006, p. 79) pero que aún registra niveles preocupantes de pobreza e inequidad.

En este marco, los avances en materia de derechos civiles y políticos no se han correspondido con avances en materia de derechos económicos, sociales y culturales. Esa relación, por muchos descuidada, recobra fuerza en el debate político recientemente y se perfila como el centro de la agenda de trabajo para la región en los próximos años. ¿Cómo entender esta indivisibilidad en el goce de estos derechos? Sin duda el tema de derechos políticos, entendidos éstos como el poder elegir y el poder ser electo, y la capacidad de los Estados de garantizar su pleno ejercicio, ha sido ampliamente analizado (Munck 2005, 2009; Bowman, Lehoucq \& Mahoney 2005; Munck y Verkuilen 2002; United Nations Development Programme 2004, 2010, 2011).

En este cuerpo de investigación, se concibe la democracia como una forma de organizar el poder en la sociedad a fin de ampliar el ejercicio de estos derechos, evitar o limitar la dominación de individuos o grupos que impidan ese objetivo y lograr la perdurabilidad de la organización democrática (PNUD 2010). 
En otras palabras, la democracia debe apuntar a reducir las desigualdades, o en último caso, mediarlas a través de la redistribución del poder a fin de que se logre el pleno ejercicio de los derechos políticos, por un lado, y por el otro, que esta acción se traduzca en políticas que beneficien a todos los sectores y actores y no solo a aquellos que, por sus capacidades económicas, o niveles de acceso a espacios de poder, puedan tener comparativamente mayores niveles de influencia política.

Los Estados Latinoamericanos han tomado medidas de política pública para generar mayor inclusión y una redistribución de los beneficios del crecimiento económico con la intención de que esto se traduzca en el goce de más derechos (en todas sus esferas ${ }^{3}$ ) para los ciudadanos y ciudadanas. Las notas distintivas de estos últimos años incluyeron la implementación de programas integrales para la reducción de la pobreza, asociados al fortalecimiento de los activos y capacidades humanas. A su vez, los pilares no contributivos de pensiones fueron una herramienta útil para incluir a los sectores informales y la ampliación del acceso a la salud permitió que poblaciones históricamente desafectadas gozasen de este derecho. Paralelamente, el rol de un mercado de trabajo robusto e inclusivo fue fundamental para asegurar medios de subsistencias dignos y legítimos.

En aras de enfrentar la desigualdad, son muchas las consideraciones de política pública que deben tomarse en cuenta. En la región, los Estados han optado por políticas que tienen como objetivo reducir la inequidad así como también por políticas fiscales, de generación de empleo y protección social. Las políticas de género y las políticas para garantizar la calidad en los servicios (educación, salud, agua, saneamiento) también han empezado a ser parte de la agenda política con más ímpetu. Para ilustrar algunas de éstas políticas, y dando cuenta de algunas de las consecuencias que el incremento de dichas inversiones en materia de política social supuso, vale la pena mencionar la tendencia en los siguientes indicadores a nivel regional.

\footnotetext{
${ }^{3}$ Tal como plantea Marshall (1997) en su obra Ciudadanía y Clase Social. Esas esferas incluyen la ciudadanía civil, ciudadanía social y ciudadanía política.
} 
Desigualdad y exclusión social como desafíos para las democracias en América Latina.

Entre el año 2002 y 2015 se registraron importantes avances en el aumento de la afiliación y la cotización a los sistemas de pensiones. El porcentaje de trabajadores cotizantes o afiliados a sistemas de pensiones aumentó del 38\% al $50 \%$. Entre los trabajadores asalariados esta cifra pasó del $53,9 \%$ al $64,7 \%$. Lo cual equivale a que unos 40 millones más de trabajadores se afiliaron al sistema de pensiones. En el mismo periodo de tiempo, el porcentaje de ocupados afiliados aumentó 12 puntos porcentuales pasando de $37,0 \%$ a $49,3 \%$. Sin embargo, el panorama cambia para los trabajadores no asalariados, de los cuales un porcentaje inferior al $18 \%$ están afiliados a un sistema de pensiones. Lo anterior teniendo en cuenta que entre el año 2002 y 2015 este porcentaje aumentó más de 8 puntos porcentuales. Dicho aumento, se ha dado, en parte, por el esfuerzo de los Estados de fomentar la incorporación voluntaria de los trabajadores independientes a los sistemas de pensiones y la expansión de las prestaciones no contributivas en la región (CEPAL, 2018b) ${ }^{4}$.

Aunque con efectos limitados para combatir la desigualdad, y con riesgos de abuso latentes, los sistemas de transferencias condicionadas han sido un instrumento utilizado para garantizar ingresos en los hogares más pobres. En los últimos años, la cobertura de los programas de transferencias condicionadas (PTCs) orientados a familias que viven en condiciones de pobreza fue ampliada en la región, pasando de menos de 300.000 hogares en 1997 a 29,8 millones en 2015. Lo cual corresponde al $17,5 \%$ del total de hogares de la región y al $20,9 \%$ de la población regional. En el año 2015, la región contaba con 30 PTCs activos en 20 países (Cecchini \& Atuesta, 2017).

Por último, en el sector salud también se han venido considerando opciones de política pública que han mejorado, aunque levemente en comparación con la necesidad y el carácter humano de este derecho, el acceso a la salud. Según la CEPAL, a inicios de la década del 2000 la afiliación al sistema de salud no superaba el $50 \%$ en los primeros siete deciles de la distribución del ingreso. Sin embargo, alrededor del 2013 se estimó una afiliación al sistema de salud de entre

\footnotetext{
${ }^{4}$ Cifras tomadas del informe: Panorama Social de América Latina, 2017, CEPAL (2018b).
} 
el $64,6 \%$ en el primer decil y el $85,5 \%$ en el décimo decil. Ahora bien, el análisis del porcentaje de afiliación a los sistemas de salud basado en las brechas de bienestar, da cuenta que en los países de brechas modestas, la afiliación a sistemas de salud del primer decil es del $68,3 \%$, en los de las brechas moderadas del $74,5 \%$ y en los de las brechas extremas de apenas un $10 \% 5$.

El efecto cumulativo del mayor crecimiento económico registrado en la región en los últimos años y la implementación de políticas de redistribución del ingreso y combate a la desigualdad habían arrojado alguna mejora en cuanto a pobreza e inequidad pero recientemente se vienen reportando retrocesos. En América Latina, la región más desigual del mundo, los niveles de pobreza y pobreza extrema aumentaron durante el año 2015 y 2016, después de que ambos fenómenos se hubieran reducido entre el año 2002 y 2014. Mientras en el año 2014 el 28,5\% de la población de la región se encontraba en situación de pobreza (168 millones de personas) en el año 2015 aumentó a 29,8\% (178 millones de personas) y en el año 2016 al 30,7\% (186 millones de personas). Por su parte, la pobreza extrema pasó del 8,2\% en 2014 al 10\% en 2016. Es decir que afectó a 13 millones de personas más en 2016 con respecto al $2014^{6}$.

En cuanto al grado de desarrollo democrático, la sociedad latinoamericana se enfrenta a tendencias que obstaculizan la garantía de los derechos y libertades, la calidad de las instituciones y la consolidación del sistema de representación política. Aunque hasta el año 2013 se hubieran dado progresos considerables, factores como el autoritarismo en los liderazgos, la corrupción, la pobreza y la marginación, han permeado la sociedad y han repercutido sobre los índices de desarrollo democrático de América Latina. Los cuales, basando el análisis en el promedio regional, cayeron un $3 \%$ en el año 2016. Dicho resultado se debe a que en términos generales y sobre la base de las cuatro dimensiones de la democracia: la de los ciudadanos, de las instituciones, la social y la económica; no se hayan materializado más avances que retrocesos.

\footnotetext{
${ }^{5}$ Cifras tomadas del informe: Panorama Social de América Latina 2015, CEPAL (2016).

${ }^{6}$ Tomado de Panorama Social de América Latina 2017, CEPAL (2018b).
} 
Desigualdad y exclusión social como desafíos para las democracias en América Latina.

Según los datos más recientes del Índice de Desarrollo Democrático de América Latina (Fundación Konrad Adenauer, 2016) han disminuido los niveles de participación en los procesos electorales, no ha habido mejoras significativas en los indicadores de respeto de los derechos políticos y las libertades civiles y se mantienen vigentes las alertas de desempleo, pobreza y desigualdad. Sin embargo, se detalla que ningún país retrocede en el puntaje de respeto de las libertades civiles y que en promedio la participación política de la mujer ha mejorado en los tres poderes del Estado ${ }^{7}$. Bajo este contexto, los países del Cono Sur siguen liderando el desarrollo democrático latinoamericano con Chile y Uruguay, mientras en la región andina el ranking lo lidera Perú y Ecuador ${ }^{8}$.

Con respecto las restricciones al derecho al voto, los avances han sido importantes: cada vez son menos los países que restringen el voto a ciertos grupos (militares en ejercicio, policías, clero, ciudadanos condenados o en espera de juicio). Hoy en día, la mayoría de los países latinoamericanos cuentan con regulaciones para permitir la emisión del voto a los ciudadanos que se encuentran en el exterior, con excepción de Nicaragua, Uruguay y Cuba. Desde el año 1990 hasta el año 2014, se aumentó de tres a quince, el número de países que diseñaron dispositivos para garantizar el derecho al voto en el exterior ${ }^{9}$. Sin embargo, la legitimidad e integridad de las elecciones en la región han sido en repetidas ocasiones cuestionadas por los ciudadanos. En las elecciones de Perú en el año 2016, hubo fuertes señalamientos a causa de los procedimientos de tachas, retiros, renuncias y exclusión de candidaturas que generaron incertidumbre hasta el momento de la elección ${ }^{10}$. En Nicaragua, las elecciones de 2016 estuvieron acompañadas de acusaciones de fraude. En la segunda vuelta de las elecciones ecuatorianas de 2017, se generaron protestas masivas después de no haber sido reconocidos los resultados por parte de la oposición. En Venezuela, los titulares asociados con el régimen chavista han sido cuestionados por

\footnotetext{
${ }^{7}$ Cifras tomadas de Fundación Konrad Adenaur (2016).

8 Tomado de Fundación Konrad Adenaur (2016).

${ }^{9}$ Tomado de PNUD (2016).

10 Según el informe Preliminar de la Misión de Observación Electoral de la OEA. Todos los informes: http://www.oas.org/es/centro noticias/comunicados prensa.asp
} 
proscribir a partidos de oposición. Lo anterior teniendo en cuenta que el gobierno canceló elecciones para elegir los gobernadores en el año 2016, lo cual fue visto como un intento para impedir que la oposición ganara más representación ${ }^{11}$.

En las cinco elecciones presidenciales que hasta la fecha se han celebrado en Latinoamérica (Costa Rica, Paraguay, Venezuela, Colombia, México), las Misiones Electorales de la OEA (MOEs) han reportado importantes avances en la garantía del voto para los adultos mayores, y para las personas con discapacidad en Costa Rica y Paraguay. Sin embargo, la representación de las mujeres sigue siendo un reto especialmente para Costa Rica donde el porcentaje de mujeres electas disminuyó con respecto al 2010, de un 38,6 a un 33,6\% en $2018^{12}$.

En Colombia se registró un aumento considerable de la participación ciudadana en parte motivado por el carácter pluralista de las candidaturas. Pese al hecho, fueron registradas amenazas en contra de algunos aspirantes, así como también la compra de votos y el proselitismo en las afueras de algunos puestos de votación. En México, las elecciones se desenvolvieron en un contexto de violencia nacional. Fue persistente el discurso ofensivo de los contendientes políticos y se vivieron casos de hurto, destrucción de boletas, balaceras y peleas alrededor de las casillas de votación. En contrapartida, México se situará entre los países de la región con mayor representación de mujeres en el congreso. En la Cámara de diputados un 49,2\% de las curules estarán ocupadas por mujeres y en el Senado esta cifra aumentará a un $50,78 \%{ }^{13}$. En Venezuela, el panorama es desalentador. En medio de la crisis humanitaria, las elecciones han sido tachadas de ilegítimas con más de 300 presos políticos en la cárcel, con candidatos y partidos proscritos, y con bajos niveles de credibilidad en las instituciones debido a la falta de garantías mínimas para los ciudadanos.

\footnotetext{
${ }^{11}$ Datos tomados del documento The Political Culture of Democracy in the Americas, 2016/17. A comparative Study of Democracy and Governance.

${ }^{12}$ Realizado con base en los Informes Preliminares de la Misión de Observación Electoral de la OEA de los países en los que se han celebrado elecciones presidenciales hasta Julio de 2018.

${ }^{13}$ Realizado con base en los Informes Preliminares de la Misión de Observación Electoral de la OEA de los países en los que se han celebrado elecciones presidenciales hasta Julio de 2018.
} 


\section{LA OTRA CARA DE LA MONEDA: DESAFÍOS EN MATERIA DE INCLUSIÓN SOCIAL Y SUS IMPLICACIONES PARA LAS DEMOCRACIAS}

Después de una contracción del PIB regional, la mejora de la demanda interna y el crecimiento de las exportaciones en América Latina y el Caribe produjeron que el crecimiento económico fuera positivo en 2017 (1,3\%). La inversión aumentó un 2,3\% y el consumo privado un 1,7\%. Además, la inflación, en América del Sur, disminuyó de $10,6 \%$ en el 2016 a 4,9\% en el 2017 y en el Caribe de $7,2 \%$ a $3,7 \%$, fuera del caso extremo de Venezuela que sufre una hiperinflación. A pesar de estos resultados, en Centroamérica y México, la inflación incrementó 2,6\% puntos porcentuales pasando de 3,4 en el 2016 a 6\% en el $2017^{14}$.

Ahora bien, los cálculos para el 2018 estiman que la tasa de crecimiento del PIB de América Latina y el Caribe será del 2,2\%. Cifra superior a la registrada en el año 2017 que fue del $1,3 \%{ }^{15}$. Al respecto, se espera que los países con tasas moderadas de crecimiento registren un mayor dinamismo que les permita entre otras cosas, avanzar en la lucha contra las brechas de la desigualdad y la inequidad. En este marco, los retos en materia inclusión social y su necesario efecto en la consolidación democrática en la región continúan y se extienden hacia los escenarios en los que es evidente la ineficiencia de la desigualdad.

En las regiones más desarrolladas, la desigualdad interna se encuentra en el nivel más alto en décadas. América Latina, por el contrario, alcanzó su mínimo histórico en términos de concentración del ingreso, pero aun así se mantiene como la región con los mayores niveles de desigualdad del mundo (CEPAL, 2018). A pesar de que entre el año 2002 y 2016, el coeficiente de Gini promedio disminuyó de 0,527 a $0,467^{16}$, el panorama de América Latina y el Caribe es preocupante si se le compara con otras regiones. De los 10 países más desiguales del mundo, ocho son de Latinoamérica y dos de África. En el primer

\footnotetext{
${ }^{14}$ Datos tomados del documento Balance Preliminar de las Economías de América Latina y el Caribe 2017. CEPAL (2018c).

${ }^{15}$ Datos tomados del documento Balance Preliminar de las Economías de América Latina y el Caribe 2017. CEPAL (2018c).

${ }^{16}$ Datos tomados del documento La ineficiencia de la Desigualdad 2018. CEPAL (2018a).
} 
lugar se encuentra Sudáfrica $(0,63)$, en segundo lugar Haití $(0,60)$ y en tercer y cuarto lugar Honduras y Colombia $(0,53)$. En general, desde el año 2012, el coeficiente de Gini promedio disminuyó de 0,527 a 0,467 aunque las tendencias hayan sido diversas entre los países de la región ${ }^{17}$.

Dentro de la medición de la desigualdad, la perspectiva centrada únicamente en los ingresos resulta reduccionista y falla en reconocer que las desigualdades en la región tienen orígenes y manifestaciones diversas y complejas. Género, edad, etnia, raza, identidad sexual, son elementos indispensables que han de ser tenidos en cuenta en cualquier análisis comprehensivo sobre la materia. Se habla de un hemisferio desigual pero también injusto (Insulza, 2014, p. 14). En efecto, más allá de la enorme diversidad adquisitiva de los ingresos de las personas, la desigualdad se deriva de la discriminación de clase, de raza, de género, de origen geográfico, de distinta capacidad física, etc., que, practicadas de manera categórica (es decir, excluyendo a todos o casi todos los miembros de un grupo), la convierten en un fenómeno multidimensional y la hacen incompatible con los ideales democráticos promovidos regionalmente (Insulza, 2014, p. 15).

La desigualdad es especialmente sufrida por las mujeres. Según un documento de trabajo del Programa de las Naciones Unidas para el Desarrollo (PNUD), ${ }^{18}$ es lamentable reconocer que en la región aun hoy en día las mujeres ganan, en promedio, el $71 \%$ del ingreso masculino, tienen tasas de desempleo urbano 1.4 veces mayores que la de los hombres, y dedican entre un $15 \%$ y un $30 \%$ más horas semanales al trabajo, dependiendo el país en cuestión. Al mismo tiempo existen más mujeres pobres que hombres pobres y aunque hay avances, por ejemplo, el porcentaje de mujeres sin ingresos propios en la región bajó de $42 \%$ en 2002 a $32 \%$ en 2011 , los logros siguen siendo frágiles e insuficientes y las brechas son aún intolerables $^{19}$. Según la CEPAL, en el año 2015 , la tasa de desempleo para las mujeres era del $8,6 \%$ en comparación con el $6,6 \%$ de los

\footnotetext{
${ }^{17}$ Datos tomados del documento La Ineficiencia de la Desigualdad 2018. CEPAL (2018a).

${ }^{18}$ PNUD (2015)

${ }^{19}$ Cifras tomadas del documento de OXFAM (2015)
} 
Desigualdad y exclusión social como desafíos para las democracias en América Latina.

hombres. Al mismo tiempo, mientras que la proporción de mujeres sin ingresos propios en el año 2016 era de un 26,7\%, para los hombres esta proporción alcanzaba un $10,6 \%$. Lo cual indica que casi un tercio de las mujeres de la región dependen de otras personas para su subsistencia ${ }^{20}$.

La falta de participación en el mercado de trabajo, el desempleo, la precariedad laboral y los menores ingresos son el resultado de factores estructurales que tienen consecuencias en las posibilidades existentes de participación política. Esto es, por un lado, una economía que no genera empleos en cantidad ni calidad suficientes y, por otro, la persistencia de patrones de división sexual del trabajo desiguales, que atribuyen a las mujeres una carga desproporcionada de tiempo dedicado al cuidado y al trabajo doméstico en detrimento de su desarrollo personal en otras áreas fuera de la maternidad y en menoscabo de las potenciales contribuciones que pueden hacer en el ámbito político.

El porcentaje de niños latinoamericanos que viven por debajo de la línea de la pobreza (US\$ 4/día) es el doble que el de los adultos: $36 \%$ y $19 \%$ respectivamente. Sus condiciones de vida se encuentran además afectadas por las enfermedades relacionadas con el agua y el saneamiento que representan la causa principal de la muerte de menores de cinco años. En el ámbito mundial, esta es una de las causas principales de la muerte de los niños. Se estima que más de 800 niños mueren de enfermedades prevenibles ocasionadas por agua, saneamiento e higiene de mala calidad ${ }^{21}$.

En la región, según la UNESCO, 70 millones de los 195 millones de niños, niñas y adolescentes viven en situación de pobreza sobre todo en las zonas urbanas y los entornos periurbanos. Esta situación afecta gravemente su esperanza de vida y repercute especialmente sobre su nivel de desarrollo en los primeros años de vida. En consecuencia, en el año 2016 se registraron 187 mil

\footnotetext{
${ }^{20}$ Observatorio de Igualdad de Género de América Latina y el Caribe. Información disponible en: https://oig.cepal.org/es/indicadores/poblacion-sin-ingresos-propios-sexo

${ }_{21}$ Cifras tomadas del artículo disponible http://www.bancomundial.org/es/news/feature/2016/06/29/ninos-america-latina-son-mas-afectadospor-pobreza
} 
muertes de niños menores de cinco años de edad en los primeros 28 días de vida. Por otro lado, se estima que 6,3 millones de inmigrantes son menores de 18 años que se ven obligados, en el mayor de los casos, a huir de la pobreza y violencia, la cual afecta cada día a 67 adolescentes que son víctimas de homicidios. Lo anterior teniendo en cuenta que lamentablemente 1,1 millones de niñas adolescentes entre los 15 y 19 años sufren de violencia sexual. Junto a esta realidad, la exclusión educativa sigue siendo un reto para los objetivos de desarrollo sostenible. Esta situación afecta actualmente a 14 millones de niños que además se ven expuestos por el cambio climático a situaciones de riesgo que ponen en juego el desarrollo de sus habilidades para el futuro. Así, se estima que 13,4 millones de niños viven en zonas de riesgo de sequía y 13,1 millones en lugares de potenciales inundaciones. A la par, se estima que 5,9 millones de niños menores de cinco años sufren desnutrición crónica y 3,7 millones padecen de sobrepeso ${ }^{22}$. El desafío es aún mayor si consideramos las diferencias geográficas: las tasas de repitencia y deserción escolar son significativamente mayores en zonas rurales, así como también el número de niños que tienen que trabajar para sobrevivir.

Paradójicamente, las desigualdades también se acentúan en el otro extremo etario, el de los adultos mayores. Según un estudio de la CEPAL y la $\mathrm{OIT}^{23}$, muchos países han revertido las políticas en esta materia aumentando la edad de jubilación y reduciendo las opciones de jubilación anticipada. En efecto, en América latina, las personas mayores deciden mantenerse activas por más tiempo en el mercado laboral, debido en gran parte a la falta de ingresos, especialmente en las mujeres. Empero, aunque en la región se han producido avances con respecto a la ampliación de la cobertura de los sistemas de pensiones contributivos y la extensión de sistemas no contributivos, en promedio un $57,7 \%$ de las personas de entre 65 y 69 años y un $51,8 \%$ de las personas de 70 años y más, no perciben una pensión dentro del régimen contributivo. En

22 Cifras tomadas de la información oficial de UNICEF disponible en: https://www.unicef.org/lac/que-hacemos

${ }^{23}$ Coyuntura Laboral en América Latina y el Caribe. La inserción laboral de las personas mayores: necesidades y opciones. CEPAL, OIT (2018). 
Desigualdad y exclusión social como desafíos para las democracias en América Latina.

consecuencia, la tasa de ocupación para el conjunto de las personas de 60 años y más alcanza el 35,4\%, para las personas de 65 a 69 el 39,3\% y para las personas de 70 años y más el 20,4\%. Esta situación es realmente alarmante si se toma en cuenta que, según HelpAge International (2015), en América Latina se produce el fenómeno más rápido de envejecimiento a nivel mundial.

La situación de las poblaciones afrodescendientes e indígenas es también alarmante. Según datos de CEPAL para América Latina y el Caribe da cuenta que en la región existen 150 millones de afrodescendientes y entre 40 y 50 millones de indígenas distribuidos a lo largo y ancho de todo el continente, de los cuales cerca de la mitad son niños, niñas y adolescentes menores de 18 años (CEPAL, 2018a). El desempleo afecta significativamente a estas poblaciones mientras que la gran mayoría de los ocupados indígenas y afrodescendientes se encuentra en sectores de baja productividad y calificación ${ }^{24}$; en América Latina y el Caribe para las mujeres indígenas y afrodescendientes el trabajo doméstico es la ocupación más importante. La brecha de ingresos entre las minorías étnicas y el resto de la población, en los países donde esa información está disponible, es de casi $40 \%{ }^{25}$, y los retornos a la educación son menores que los que recibe el resto de la población (Camacho, 2014: p. 264-265). Se estima que los pueblos indígenas constituyen aproximadamente el $14 \%$ de las personas pobres y el $17 \%$ de las personas extremadamente pobres en la región ${ }^{26}$. Además, como consecuencia del racismo y la discriminación, estos pueblos tienen niveles nutricionales más bajos, menor cobertura y calidad en los servicios educativos, y un limitado o inexistente acceso a otros servicios básicos como salud, agua, saneamiento y protección social.

\section{UNOS MÁS IGUALES QUE OTROS: IMPLICACIONES DE LA DESIGUALDAD PARA LA DEMOCRACIA}

\footnotetext{
${ }^{24}$ La situación de las personas afrodescendientes en las Américas, OEA. (CIDH, 2011).

25 Ñopo, Hugo. (2012), New Century Old Disparities, gender and ethnic earnings gaps in Latin America and the Caribbean, BID.

${ }^{26}$ Latinoamérica Indígena en el siglo XXI. Primera década. (Banco Mundial, 2015).
} 
Ante este panorama de desigualdad, es pertinente preguntarse cómo se traducen estos retos a nivel político y qué implicancias tiene esta fotografía social para la democracia. Un reto cada vez más documentado tiene que ver con el desencanto de la ciudadanía con la democracia, y vinculado a esto, la erosión de la legitimidad del estado. Azpuruh \& Smith (2012, p. 30) señala en un estudio de opinión que en general en las Américas, los ciudadanos están de acuerdo con que el Estado se involucre en la reducción de la desigualdad, y al mismo tiempo la desigualdad persiste teniendo implicancias importantes para el funcionamiento democrático. Otros efectos de la persistencia de sociedades excluyentes se vinculan al aumento de la violencia y otras prácticas antisociales así como el acceso diferenciado a derechos básicos como la justicia o la participación política, en particular para poblaciones indígenas y afrodescendientes. ${ }^{27}$ Finalmente, en esta sección, también se evalúa la nueva conflictividad que emerge de las clases medias como consecuencia de las inequidades socioeconómicas, predominantemente por los llamados "strugglers" ${ }^{28}$ así como la réplica de las asimetrías socioeconómicas en asimetrías sociopolíticas, a la luz de las consecuencias que esto tiene para la representación y calidad de la toma de decisiones.

\section{a) El desencanto de la ciudadanía con la democracia, y erosión de su legitimidad}

Según un estudio de la CEPAL (2012), hay una fuerte correlación entre las apreciaciones de injusticia y desigualdad por un lado, y la desconfianza de los ciudadanos y ciudadanas en las instituciones tradicionales (partidos políticos, poderes ejecutivos, legislativos y judiciales) por el otro. Esto indica la persistencia de un profundo malestar ciudadano con el modo en que funcionan las instituciones

\footnotetext{
${ }^{27}$ Desigualdad e Inclusión Social en las Américas. (OEA, 2014).

${ }^{28}$ En este punto vale resaltar la situación de un tercio de la población total de América Latina que, según un estudio de Birdsall et al (2014), vive con un ingreso de entre 4 y 10 dólares diarios. Los llamados por estos autores "luchadores" (strugglers) incluyen a sectores de la población que han superado la pobreza que aún aqueja a más de 167 millones de latinoamericanos. Sin embargo, Insulza $(2014,14)$ advierte del riesgo de considerarlos parte de "sectores medios" ya que estos "no pobres" se ubican en una zona de ingreso que los hace aun extremadamente vulnerables.
} 
Desigualdad y exclusión social como desafíos para las democracias en América Latina.

y se distribuyen los bienes económicos, sociales, y políticos en los países Latinoamericanos. A fin de cuentas, una democracia sin bienestar eventualmente va socavando la percepción que los ciudadanos tienen sobre dicho sistema, menoscabando su legitimidad y utilidad (Zovatto \& Herrera, 2011).

Según el informe de la Corporación Latinobarómetro del año 2017, por quinto año consecutivo la percepción sobre la democracia en América Latina no mejora. Con respecto al año 2016, el porcentaje de apoyo a la democracia bajó un punto y se ubicó en 53\%. Incluso, con el periodo de crecimiento económico que se vivió entre el 2016 y el 2017. Así mismo, solo un 5\% de los ciudadanos expresa que existe plena democracia, con respecto al $72 \%$ que expresa que la democracia enfrenta graves problemas. Además, la satisfacción con la democracia disminuyó de un $34 \%$ en el 2016 a un $30 \%$ al $2017^{29}$.

En este sentido, Azpuru y Smith (2012) dan cuenta cómo el pertenecer a uno de los grupos generalmente discriminados (ser mujer, indígena o tener piel oscura) afecta el apoyo al sistema político y el apoyo hacia la democracia (Azpuru y Smith 2012, p. 75). Igualmente, las discriminaciones a nivel social también se traducen en límites a las oportunidades de acceder al sistema político. Con frecuencia entonces son pocos los que acceden al poder político, y son pocos los que cuentan con los fondos para ingresar a la política y armar sus campañas. El electorado, por su parte, está cada vez más polarizado ideológicamente. La proporción de encuestados que se hacen llamar de centro en lugar de izquierda o de derecha se ha reducido del $42 \%$ en 2008 , al $33 \%$ en 2015. En este sentido, y como bien demuestran Pérez-Liñan y Mainwaring (2008), la desigualdad y exclusión social se relacionan directamente con el desencanto democrático, que a su vez se consolida dada la recurrente debilidad de los mecanismos de control del poder político existente. Se perfila, entonces, una hipótesis a ser evaluada de que "a mayor desigualdad, mayor es el desencanto con las instituciones democráticas, y con la democracia en general." Las percepciones ciudadanas así parecen indicarlo.

\footnotetext{
${ }^{29}$ Informe Latinobarometro 2017.
} 


\section{b) Aumento de la violencia y otras prácticas antisociales}

El Banco Mundial ha venido estudiando la relación entre desigualdad y violencia. La violencia es sin duda otra consecuencia de la desigualdad que afecta en un sentido más directo, a la seguridad del ciudadano común, y en un sentido amplio, cuestiona la viabilidad de un sistema democrático. En Septiembre de 2014, el Banco Mundial publicó un informe que muestra como una distribución del ingreso más inequitativa ha contribuido a aumentar la violencia y las tasas de crimen en dos mil municipalidades mexicanas. ${ }^{30}$ Por un lado, la desigualdad genera una sensación de injusticia entre las personas en desventaja que les lleva a buscar una compensación por otros medios, incluyendo actividades criminales. Por otro lado, la actividad criminal también se puede explicar por un análisis costobeneficio. En este sentido, cuanto más escasas sean las oportunidades económicas para los sectores pobres y mayor sea la brecha de ingreso entre clases, los beneficios económicos de crímenes como robos o secuestros -que muchas veces terminan en homicidios- tienden a ser mayores. Un detalle interesante es que la desigualdad provoca un aumento del crimen incluso cuando se comparan municipalidades que han tenido la misma evolución de la tasa de pobreza. Es decir, un aumento en la desigualdad debido a que los ricos se vuelven más ricos (y no a un aumento en el porcentaje de pobres) es lo que ha contribuido a incrementar la tasa de homicidios en algunos municipios de México ${ }^{31}$.

En esta misma línea, un estudio del Banco Interamericano de Desarrollo ${ }^{32}$ ha demostrado para nueve países de la región que aquellos trabajadores que no perciben los beneficios de estar incluidos en el mercado laboral formal (negociación colectiva, vacaciones, aguinaldo, seguro médico) tienen una imagen negativa no solo de sus empleadores sino del Estado en su conjunto. Esos mismos empleados consideran que este último no ha protegido sus derechos, y

\footnotetext{
30 Información tomada del artículo del Banco Mundial (2014) "Está demostrado: con menos desigualdad se tiene menos crimen".

31 "Income Inequality and Violent Crime. Evidence from Mexico's Drug War". Banco Mundial (2014)

${ }^{32}$ La exclusión laboral y la erosión de las responsabilidades ciudadanas (BID, 2015).
} 
Desigualdad y exclusión social como desafíos para las democracias en América Latina.

por ende sienten menos obligaciones de respetar sus obligaciones ciudadanas (obedecer la ley, pagar impuestos, y votar) (Ronconi \& Zarazaga, 2015).

\section{c) Acceso diferenciado a derechos, especialmente para indígenas y afrodescendientes ${ }^{33}$}

Una democracia sin estado de derecho, sin garantías básicas de acceso a la justicia es una democracia en déficit. En sociedades excluyentes, los sectores más pobres, siendo en el caso latinoamericano los indígenas y afrodescendientes, no gozan de ese derecho en igualdad de condiciones. Las poblaciones afrodescendientes e indígenas enfrentan barreras para participar en democracia, en la medida en que, abrumadoramente, forman parte de los estratos socio económicos de más bajos ingresos. En efecto, la evidencia sugiere que los afrodescendientes se encuentran "desproporcionalmente en las áreas residenciales más pobres" y "padece(n) una situación de discriminación estructural." ${ }^{34}$ El $90 \%$ de las poblaciones afrodescendientes en los países de la región viven en pobreza y pobreza extrema, y en muchos casos no gozan de acceso universal a los servicios de salud, educación, vivienda y agua potable. ${ }^{35}$ Lo mismo se aplica a los pueblos indígenas en la región. A pesar de que en los últimos veinte años los grupos de indígenas han avanzado considerablemente hacia una mayor inclusión en el sistema democrático en su derecho de elegir y ser electo continúan enfrentando importantes desventajas para su participación política (Casas-Zamora et al, 2014).

En el acceso a la justicia se replica un patrón similar. Por ejemplo, los índices actuales sobre la situación de las personas pertenecientes a los pueblos indígenas en las prisiones reflejan los desafíos que impone el tema del acceso a la justicia para este sector de la población. En ciertos países la sobrerrepresentación

\footnotetext{
${ }^{33}$ Desigualdad e Inclusión Social en las Américas (OEA, 2014)

${ }^{34}$ La situación de las personas afrodescendientes en las Américas (CIDH, 2011).

${ }^{35}$ Mala Htun (2014). Political Inclusion and Representation of Afrodescendant Women in Latin America. Por ejemplo, 43\% de afrodescendientes viven bajo de la línea de pobreza en Brasil, comparado con $22 \%$ de blancos. Con respeto al acceso a la educación, el logro educativo promedio de un afrodescendiente de Brasil en 2006 fue de 6,2 años (un aumento de 4,3 años en 1995).
} 
de indígenas en las cárceles y las paupérrimas condiciones en que se hallan estas personas detenidas, viola incluso principios reconocidos internacionalmente. Un estudio presentado por el Mecanismo de Expertos de las Naciones Unidas en el año 2013 confirma dichos datos, señalando que en algunos países la población indígena en las cárceles es cinco veces mayor al porcentaje de detenidos de otros sectores de la ciudadanía (ACNUDH, 2013). Para el Relator Especial de Naciones Unidas sobre los Pueblos Indígenas, la excesiva presencia de indígenas en las cárceles encuentra su explicación, en muchos casos, en el uso de lenguas no indígenas durante el proceso judicial, sin la presencia de intérpretes o defensores indígenas de oficio o frente a funcionarios con prejuicios o estereotipos negativos contra ellos.

Por su parte, los afrodescendientes han sido durante siglos víctimas de la esclavitud, la discriminación racial y la denegación de sus derechos fundamentales, entre ellos, el pleno acceso a la justicia. Según el estudio "Sistema Judicial y Racismo contra Afro-descendientes: Brasil, Colombia, Perú y República Dominicana", realizado por el CEJA en marzo de 2004, la persecución penal en nuestro continente es más alta sobre las personas afrodescendientes que sobre las personas de otras razas. Estadísticamente se han registrado muchos casos de afrodescendientes que han sido condenados injustamente (Gonzales \& Contesse, 2004). Esta situación se agrava cuando estamos ante jóvenes y mujeres afrodescendientes. Esto se debe a una estigmatización histórica en su condición de acusadas o culpables de actos delictivos por el solo hecho de pertenecer a este colectivo. Otro tema de no menor importancia es el número ínfimo de afrodescendientes que son funcionarios en los poderes judiciales de las Américas, sobre todo en América Latina. En efecto, los mayores resultados de las políticas de inclusión de los afrodescendientes en el aparataje público se han producido en especial a nivel de los poderes ejecutivo y legislativo, siendo muy incipiente en el poder judicial. En suma, el resultado de esta situación de exclusión es una democracia con sesgos fuertes traduciéndose en las ya mencionadas “democracias de baja calidad” O'Donnell (1998). 
Desigualdad y exclusión social como desafíos para las democracias en América Latina.

\section{d) Nueva conflictividad: La rebelión de las clases medias}

Está muy bien documentado el hecho de que el crecimiento económico en la región ha producido el ensanchamiento de la clase media ${ }^{36}$, y consecuentemente el aumento de las demandas ciudadanas. Esto hace que la desigualdad sea vivida cada vez con mayor hastío. La expansión de la clase media ha traído transformaciones políticas importantes y una redistribución ya no solo de ingreso, sino de poder, en su favor. Una América Latina donde la clase media es mayoría, es una región donde está siendo cada vez más potente la exigencia de bienes y servicios públicos de calidad, y de acceso a derechos fundamentales. Es también una región en la que muchas familias, que por primera vez tienen acceso a una vivienda digna, a un automóvil y a un crédito bancario, harán valer todo su poder para que el gobierno no haga despropósitos con los equilibrios macroeconómicos (Casas-Zamora et al, 2014, p. 70-71).

En ese sentido, la percepción de la desigualdad mencionada anteriormente aunada a una mejora en las condiciones socio-económicas en esta nueva clase media, a riesgo de bajar por debajo de la línea de la pobreza, crea un caldo de cultivo para la conflictividad social. Así lo demuestran hechos de este último ciclo en la región. En Chile se constataron protestas con la exigencia de acceso a una educación gratuita de calidad. En Brasil también se vieron protestas masivas, y hasta violentas como resultado del incremento en las tarifas de transporte en la penúltima Copa Mundial de Fútbol celebrada en ese país.

El acceso desigual a la educación, a los servicios públicos, y en muchos casos un descontento con las élites políticas y la corrupción son, con cada vez más frecuencia, la raíz de estas protestas, que afectan directamente la gobernabilidad de los países en donde han ocurrido. El progreso económico y social, y la expansión de la clase media ayudó a sacar a muchos de la pobreza, y elevó el piso de protección de muchos, pero a la vez, elevó las expectativas del desempeño del sistema democrático, elevó también las demandas por aun más

36 Banco Mundial, Shifting Gears to Accelerate Shared Prosperity in Latin America and the Caribbean, World Bank Document 78507 (2013). 
garantías para ejercer plenamente derechos y libertades, y empoderó a las masas de cuestionar la priorización del gasto público.

\section{e) Asimetría Social-Asimetría Política: representatividad y calidad de la toma de decisiones}

¿Qué pasa cuando son pocos los privilegiados? ¿Qué pasa cuando solo acceden al poder aquellos con acceso a fondos para ingresar a la política y armar sus campañas?, ¿Qué pasa cuando aquellos con acceso a redes de financiamiento y de contactos son los únicos que acceden al poder? El campo político revela o más bien replica las asimetrías de poder que se dan en el ámbito socio-económico. La participación de mujeres en espacios de poder es de las asimetrías más estudiadas, pero es vital también considerar las asimetrías en la participación de los sectores más pobres y vulnerables. El llamado es a atender este tema ya que las brechas de inequidad, de no ser atendidas, corren el riesgo de ser replicadas inter-generacionalmente en la región perpetuando, de esta forma, el fenómeno.

Un estudio (2013) producido por PNUD titulado "Ciudadanía Intercultural" 37 documenta la realidad de las minorías étnicas en las Américas. Los datos hablan del permanente estado de desigualdad socio-económica pero también política que experimentan. Poniendo la lupa en la participación de las comunidades indígenas, según cifras de la ONU, en América Latina y el Caribe los pueblos indígenas tienen una población de unos 50 millones de personas (cerca del 10\% de la población). Mientras en Perú y Guatemala los indígenas son casi la mitad de la población y en Bolivia superan el $60 \%$, es México quien tiene la mayor población indígena de la región en términos absolutos con 25’694,928 millones de personas $(21,5 \% \text { de su población total })^{38}$. Sin embargo, las asimetrías en la representación política son abrumadoras. Se citan dos casos, el de México que con una cámara de 500 diputados, para el periodo del 2012-2015 solo tenía 14 diputados indígenas

${ }^{37}$ Ciudadanía Intercultural: Aportes desde la participación política de los pueblos indígenas de Latinoamérica (PNUD, 2013).

${ }_{38}$ En concordancia con los indicadores socioeconómicos de los Pueblos Indígenas de México 2015. Información recopilada del Informe Preliminar de la MOE en las elecciones de México 2018. 
Desigualdad y exclusión social como desafíos para las democracias en América Latina.

(2.8\%) y solo cuatro de los diputados indígenas eran mujeres $(0.8 \%)$, o el de Guatemala que con un total de 158 curules, que en el periodo del 2012-2015 solo contaba con 19 indígenas (12\%) y tres mujeres indígenas $(1.89 \%)$ otros casos similares: Nicaragua, Ecuador, Perú, Bolivia). En México para el año 2018, por disposición del Instituto Nacional Electoral (INE) debieron postularse candidaturas indígenas en la elección de diputaciones por el principio de mayoría relativa en los treces distritos uninominales en los que esta población supera el $60 \%$ de la población. Este hecho indica avances en el establecimiento de medidas de acción afirmativas para favorecer los niveles de participación de la población indígena en la región.

Como bien describe Rangel (2005a), la realidad de la comunidad afrodescendiente es similar. Tal como se ha mencionado, el $90 \%$ de estas poblaciones en los países de la región viven en pobreza y pobreza extrema, y en muchos casos no gozan de acceso universal a los servicios de salud, educación, vivienda, agua potable. ${ }^{39}$ Esto crea condiciones para que se perpetúe el fenómeno de la subrepresentación política en los puestos de toma de decisión, desde la oferta hasta el ejercicio del poder, traduciéndose esto en la formulación de políticas públicas que no consideran la especificidad étnica de la población (Rangel, 2005b).

La principal consecuencia de esta desigualdad que es primordialmente social pero que se replica a nivel político es niveles bajos de representatividad y calidad de las decisiones que emanan del sistema político, y que llevan de nuevo a la problemática del desencanto con la democracia y la conflictividad social. Estas asimetrías sociales y políticas afectan la formulación de políticas públicas, y afectan la priorización del gasto público que con frecuencia para las mayorías ha venido siendo equivocadas, tras varias décadas de gobiernos elegidos popular y democráticamente.

\footnotetext{
${ }^{39}$ Political Inclusion and Representation of Afrodescendant Women in Latin America, (2014). Por ejemplo, $43 \%$ de afrodescendientes viven bajo de la línea de pobreza en Brasil, comparado con $22 \%$ de blancos. Con respeto al acceso a la educación, el logro educativo promedio de un afrodescendiente de Brasil en 2006 fue de 6,2 años (un aumento de 4,3 años en 1995). Para los de tez blanca, las cifras son 8,0 y 6,4, respectivamente. Esto implica una diferencia de 1,8 años en 2006 (Pp. 12-13).
} 


\section{SOCIEDADES QUE INCLUYEN, DEMOCRACIAS QUE SE CONSOLIDAN}

Los dilemas que enfrentan las democracias de América Latina sin duda son muchos. Este trabajo priorizó la vinculación entre la viabilidad socioeconómica de las democracias. Los déficits en materia de inclusión social condicionan cada vez de forma más evidente, la calidad de estas democracias. Este trabajo argumenta que sin duda el crecimiento económico reciente ha sido importante en esta región, pero al mismo tiempo se considera como impostergable el logro de avances en cuanto a la plena inclusión de todos los ciudadanos y ciudadanas en los beneficios de ese crecimiento económico.

En el debate teórico y práctico, se viene sosteniendo con más frecuencia que además de las debilidades que aún existen en nuestras instituciones y en nuestra práctica política, la plena vigencia de la democracia en América adolece de un grave problema de desigualdad, que no solo afecta a la convivencia democrática, sino que es también un obstáculo para el crecimiento sano. Se ha tomado conciencia que la enorme injusticia que existe en nuestros países en la distribución de la riqueza y en el acceso a los bienes sociales daña gravemente el tejido democrático. Por ello, el debate ha dejado de ser puramente económico y se ha trasladado al campo de las políticas públicas. Las decisiones políticas que han tomado (y que han de tomar) los Estados para mejorar la distribución es lo que hace compatible la economía de mercado con la democracia, y a ellos corresponde encontrar un adecuado equilibrio, en el marco del Estado de derecho y de la división de poderes, entre el crecimiento y la reducción de la desigualdad. La gran deuda de la región en términos de la pobreza, la desigualdad y la exclusión social, así como respecto a la demanda ciudadana por una igualdad económica, política y social, apuntan hacia la necesidad de dar respuestas hoy más que nunca.

En la jurisprudencia interamericana, la academia, en los propios debates que se dan en el marco político regional existe el consenso que establece que los derechos humanos son complementarios, interdependientes e indivisibles. Es decir, no existen jerarquías, no es válido priorizar el logro de unos versus otros. 
Desigualdad y exclusión social como desafíos para las democracias en América Latina.

Los ciudadanos tienen la expectativa de que los estados deben trabajar para garantizar el ejercicio pleno de todos los derechos. A tres décadas de las transiciones democráticas, sin duda hemos avanzado mucho en el logro del ejercicio de derechos civiles y políticos, sin embargo, aún estamos rezagados en lo relativo a los derechos económicos, sociales y culturales. Estos que tienen que ver con la equidad y la inclusión social.

El informe de la OEA y PNUD (2010) titulado "Nuestra Democracia" señala la visión del desarrollo económico y la inclusión social como un fin de la democracia, priorizando la relación interdependiente entre lo político y lo económico, es decir, priorizando la necesidad de garantizar la viabilidad política de los programas económicos que permiten el goce de los DESC pero también la viabilidad económica de la democracia ${ }^{40}$. Podemos incluso argumentar, que a mayor goce de los derechos económicos, sociales y los beneficios de la cultura, mayor gobernabilidad y viabilidad democrática habrá en los países del Hemisferio. Esta sin duda es la agenda pendiente que tiene la clase política con su ciudadanía.

\section{REFERENCIAS BIBLIOGRÁFICAS}

ACNUDH - Alto Comisionado de Derechos Humanos. (2013). Los Pueblos Indígenas y el Sistema de Derechos Humanos de las Naciones Unidas. Folleto informativo № 9/Rev.2. (Pp.1-48). Extraído de: https://www.ohchr.org/documents/publications/fs9rev.2 sp.pdf. Consulta: 26/07/2018.

Azpuru, Dinorah y Amy Érica Smith. (2012). El efecto de la desigualdad de oportunidades y la discriminación en la legitimidad política y la participación. En Azpuru, Dinorah y Pira, Juan Pablo (Eds.) Cultura política de la democracia en Guatemala y en las Américas. United States Agency for International Development (USAID). Estados Unidos.

Banco Mundial (2015). Latinoamérica Indígena en el siglo XXI. Primera década. Extraído http://documents.worldbank.org/curated/en/541651467999959129/pdf/9854

\footnotetext{
40 Informe "Nuestra Democracia" de Programa de las Naciones Unidas para el Desarrollo, Secretaría General de la Organización de los Estados Americanos. - México : FCE,PNUD, OEA, 2010
} 
4-WP-P148348-Box394854B-PUBLIC-Latinoamerica-indigena-

SPANISH.pdf Consulta: 11/07/2018.

Banco Mundial. (2014). Está demostrado: con menos desigualdad se tiene menos

crimen.

Extraído

de:

http://documents. worldbank.org/curated/en/236161468299090847/pdf/WPS

6935.pdf. Consulta: 28/07/2018.

BID. (2015). La exclusión laboral y la erosión de las responsabilidades ciudadanas. Extraído de:

https://publications.iadb.org/handle/11319/6828?locale-attribute=en Consulta: 11/07/2018.

Birdsall, Nancy; Lustig, Nora \& Mayer, Christian. (2014). The Strugglers: the New Poor in Latin America. Centre for Global Development. Vol. 60.No 337. Extraído de: https://www.cgdev.org/sites/default/files/new-poor-latinamerica 1.pdf Consulta: 11/07/2018.

Bowman, Kirk, Fabrice Lehoucq \& James Mahoney. 2005. "Measuring Political Democracy: Case Expertise, Data Adequacy, and Central America", Comparative Political Studies, Vol. 38, No. 8: 939-70. Estados Unidos (Pp. 939-970).

Camacho, María Claudia. (2014). Desigualdad en el empleo y el trabajo en Desigualdad e Inclusión Social en las Américas: 14 Ensayos. 2014. Organización de los Estados Americanos. Estados Unidos.

Casas-Zamora Kevin, Muñoz-Pogossian Betilde \& Vidaurri, Marian (2014). Consideraciones sobre la desigualdad política en las Américas. En Insulza, José Miguel. Desigualdad e Inclusión Social en las Américas: 14 Ensayos (2014). Organización de los Estados Americanos, Estados Unidos.

Cecchini, Simone y Atuesta, Bernardo. (2017). Programa de Transferencias condicionadas en América Latina y el Caribe. Serie Políticas Sociales. No 224. (Pp. 1-84). Extraído de: https://repositorio.cepal.org/bitstream/handle/11362/41811/1/S1700419 es. pdf Consulta: 11/07/2018.

CEPAL (2012). Panorama Social de América Latina 2012. Extraído de: https://repositorio.cepal.org/bitstream/handle/11362/1247/1/S2012959 es.pdf Consulta: 11/07/2018.

CEPAL. (2016). Panorama Social de América Latina 2015. Extraído de: https://repositorio.cepal.org/bitstream/handle/11362/39965/S1600175 es.pdf. Consulta: 11/07/2018.

CEPAL (2018a). La ineficiencia de la Desigualdad 2018. Extraído de: https://repositorio.cepal.org/bitstream/handle/11362/43442/6/S1800059 es.p df Consulta: 11/07/2018.

CEPAL. (2018b). Panorama Social de América Latina 2017. Extraído de: https://repositorio.cepal.org/bitstream/handle/11362/42716/7/S1800002 es.p df Consulta: 11/07/2018. 
Desigualdad y exclusión social como desafíos para las democracias en América Latina.

CEPAL (2018c). Balance Preliminar de las Economías de América Latina y el Caribe 2017.2 Extraído de: https://repositorio.cepal.org/bitstream/handle/11362/42651/117/S1701283 es .pdf Consulta: 11/07/2018.

CEPAL, OIT. (2018). La inserción laboral de las personas mayores: necesidades y opciones. Coyuntura Laboral en América Latina y el Caribe. No. 18. (Pp. 338). Extraído de: http://www.ilo.org/wcmsp5/groups/public/---americas/---rolima/---sro-santiago/documents/publication/wcms 630073.pdf. Consulta: $11 / 07 / 2018$.

Cohen, Mollie J., Lupu, Noam y Zechmeister, Elizabeth J. (2017). The Political Culture of Democracy in the Americas, 2016/17: A Comparative Study of Democracy and Governance. United States Agency for International Development. Extraído de: https://www.vanderbilt.edu/lapop/ab2016/AB201617 Comparative Report English V2 FINAL 090117 W.pdf. Consulta: $11 / 07 / 2018$.

Comisión Interamericana de Derechos Humanos CIDH. (2011). La situación de las personas afrodescendientes en las Américas. Extraído de: http://www.acnur.org/fileadmin/Documentos/BDL/2012/8311.pdf Consulta: $11 / 07 / 2018$.

Corporación Latinobarómetro. (2017). Informe 2017. Extraído de: http://www.latinobarometro.org/latNewsShow.jsp. Consulta: 11/07/2018.

Fundación Konrad Adenauer. (2016). Índice de Desarrollo Democrático de América Latina. Extraído de: http://idd-lat.org/2016/downloads/idd-lat2016.pdf?nocache $=7687652837$. Consulta: 11/07/2018.

Gonzales, Felipe \& Contesse, Jorge. (2004). Sistema Judicial y racismo contra afrodescendientes: Brasil, Colombia, Perú, y Republica Dominicana. Centro de Estudios de Justicia de las Américas. Chile. Extraído de: http://biblioteca.cejamericas.org/bitstream/handle/2015/656/raz-sistema-judracismo2.pdf?sequence=1\&isAllowed=y Consulta: 11/07/2018.

HelpAge International (2015). Índice de Envejecimiento Global. Extraído de: http://www.helpage.es/silo/files/gawi-2015-resumen-ejecutivo-.pdf. Consulta: $07 / 26 / 2018$.

Htun, Mala. (2014). Political Inclusion and Representation of Afrodescendant Women in Latin America. En Escobar, M \& Taylor, M. Representation: The case of women. Oxford University Press. Estados Unidos.

International Bank for Reconstruction and Development/ The World Bank (2013). Shifting Gears to Accelerate Shared Prosperity in Latin America and the Caribbean. Extraído de: https://openknowledge.worldbank.org/bitstream/handle/10986/15265/LAC\%2 0Poverty\%20and\%20Labor\%20Brief\%20June\%202013.pdf?sequence $=1$ Consulta: $11 / 07 / 2018$.

Insulza, José Miguel. (2014). Desigualdad, Democracia e Inclusión Social. en Desigualdad e Inclusión Social en las Américas: 14 Ensayos. Organización de los Estados Americanos, Estados Unidos. 
Marshall, Thomas Humphrey (1997). Ciudadanía y Clase social. Reis: Revista Española de Investigaciones Sociológicas. No. 79. España (Pp. 297-344). Extraído de http://catedras.fsoc.uba.ar/isuani/marshall.pdf. Consulta: $11 / 07 / 2018$.

Munck, Gerardo L. (2009). Measuring Democracy: A Bridge between Scholarship and Politics. John Hopkins University Press. Estados Unidos.

Munck, Gerardo L. (2005). "Measuring Democratic Governance: Central Tasks and Basic Problems," pp. 427-59, in Deepa Narayan, ed., Measuring Empowerment: Cross- Disciplinary Perspectives. World Bank. Estados Unidos.

Munck, Gerardo y Verkuilen, Jay. (2002).Conceptualizing and Measuring Democracy: Evaluating Alternative Indices. Comparative Political Studies, Vol. 35 No. 1. Estados Unidos (Pp.5-34).

Ñopo, Hugo. (2012). New Century Old Disparities, gender and ethnic earnings gaps in Latin America and the Caribbean, IADB. Banco Interamericano de Desarrollo BID. Washington. Extraído de: https://openknowledge.worldbank.org/bitstream/handle/10986/11953/724610 PUB0Publ067926B09780821386866.pdf? sequence $=1$ \&isAllowed $=y$ Consulta: 11/07/2018.

O' Donnell, Guillermo. (1998). Poverty and Inequality in Latin America: Some Political Reflections en Tokman, Victor E y O'Donnell, Guillermo (Editores). Poverty and Inequality in Latin America: Issues and New Challenges. University of Notre Dame Press, Estados Unidos.

OEA, PNUD (2010). Nuestra Democracia. Extracto de https://www.oas.org/es/sap/docs/nuestra dem s.pdf. Consulta: 11/07/2018.

OXFAM. (2015). Privilegios que niegan derechos. Desigualdad extrema y secuestro de la Democracia en América Latina y el Caribe. Extraído de: https://www.oxfam.org/sites/www.oxfam.org/files/file attachments/reporte igu ales-oxfambr.pdf .Consulta: 11/07/2018.

Pérez-Liñan y Mainwaring (2008). Regime Legacies and Democratization: Explaining Variance in the Level of Democracy in Latin America, 1978-2004. Working Paper \#354. Extraído de: https://kellogg.nd.edu/sites/default/files/old files/documents/354 0.pdf Consulta: 07/26/2018.

Programa de las Naciones Unidas para el Desarrollo, Secretaría General de la Organización de los Estados Americanos. (2010). Nuestra democracia. 2010. México.

Programa de las Naciones Unidas para el Desarrollo. (2013). Ciudadanía Intercultural: Aportes desde la participación política de los pueblos indígenas de Latinoamérica. Extraído de: http://www.undp.org/content/undp/es/home/librarypage/democraticgovernance/Ciudadania-Intercultural-Aportes-desde-la-participacion-politicade-los-pueblos-indigenas-de-Latinoamerica.html Consulta: 11/07/2018. 
Desigualdad y exclusión social como desafíos para las democracias en América Latina.

Programa de las Naciones Unidas para el Desarrollo. (2015). Pobreza, oportunidades económicas desiguales y género: hipótesis para la discusión. Extraído de: http://www.py.undp.org/content/dam/paraguay/docs/documento\%20de\%20tra bajo\%20COMPLETO.pdf Consulta: 11/07/2018.

Programa de las Naciones Unidas para el Desarrollo (PNUD) (2016). Estudios Electorales en Perspectiva Internacional Comparada. El voto en el extranjero en 18 países de América Latina. Extraído de: http://portal.ine.mx/wpcontent/uploads/2017/04/Votoex AL.pdf Consulta: 11/07/2018.

Rangel, Marta. (2005a). La población afrodescendiente en América Latina y los Objetivos de Desarrollo del Milenio. Un examen exploratorio en países seleccionados utilizando información censal. CEPAL. Extraído de: http://repositorio.cepal.org/bitstream/handle/11362/4054/S2006017 es.pdf?s equence=1 Consulta: 07/28/2018.

Rangel, Marta. (2005b). Propuestas para el análisis comparado de temas destacados de los derechos humanos de los afrodescendientes en América Latina. Serie Población y Desarrollo No. 59. (Pp. 3-63) Extraído de: https://repositorio.cepal.org/bitstream/handle/11362/7201/1/S05835 es.pdf Consulta: 11/07/2018.

Ronconi, Lucas \& Zarazaga. Rodrigo. (2015). Labor Exclusion and the Erosion of Citizenship Responsibilities. IDB Working Paper Series No. IDB-WP-556. Inter-American Development Bank, Department of Research and Chief Economist, Estados Unidos.

Wehr, Ingrid. (2008). Desigualdad y Democracia. Nueva Sociedad, No. 215. Argentina (Pp. 79.94).

Zovatto, Daniel \& Marco Herrera. (2011). Diálogo sobre Democracia, Desarrollo, Cohesión Social y Seguridad en América Latina. San José, IDEA Internacional, Costa Rica. 